\title{
ML GUIDED ENERGY-PERFORMANCE TRADE-OFF ESTIMATION FOR UNCORE FREQUENCY SCALING
}

\author{
Solomon Abera Bekele \\ M Balakrishnan \\ Anshul Kumar \\ Department of Computer Science and Engineering \\ Indian Institute of Technology Delhi \\ New Delhi, India \\ \{solomon,mbala,anshul\}@cse.iitd.ac.in
}

\begin{abstract}
Chip multiprocessors (CMPs) - also called multicores - have become the main architectural component for computing systems ranging from high-end servers to hand-held devices. CMPs enhance performance through parallelism by permitting multi-programmed/threaded workloads to run concurrently on the available computing cores. However, the power-performance trade-off due to frequency scaling of these cores cannot be determined independently as they share critical resources like L2/L3 cache as well as memory during execution. Thus, unlike the uni-processor environment, the energy consumption of an application running on a CMP depends not only on its characteristics but also its co-runners (applications running on other cores). In this work, we investigate an application's performance response to core and uncore frequency scaling and propose a learning-based model for determining a suitable uncore frequency. The model takes selected individual characteristics of the applications as input and suggests an optimal uncore frequency that would satisfy the overall QoS requirements.
\end{abstract}

Keywords: CMP, Resource Contention, DVFS, Uncore Frequency Scaling (UFS), Machine Learning (ML).

\section{INTRODUCTION}

Energy efficiency is a key concern in both server class systems used in compute clusters as well as in computing cores used in hand-held devices. Power consumption constitutes the most significant operating cost for computing clouds (Sharma et al. 2016). In hand-held devices like mobiles, the number of hours a device can be used before requiring re-charge continues to be an important user concern due to limited energy available in the device. To control and reduce power consumption of processors, various techniques are in use. Dynamic Voltage and Frequency Scaling or DVFS is a popular power management technique where the frequency and associated voltage of a processor are dynamically adjusted as per the requirements to achieve higher energy efficiency. As typically lower frequency implies lower performance, energy efficiency revolves around the power-performance trade-off associated with different DVFS frequencies. Workloads that show memory-bound behavior are candidates for operating at a lower frequency as their performance is not directly determined by the speed of the core; rather it is determined by the bus bandwidth available for their transactions. In such cases switching cores at a high frequency with the associated higher voltage amounts to wasted power.

Another development in the last two decades is the wide-spread use of CMPs. In CMP, the performance that each of the computing cores can deliver cannot be independently determined as they share critical resources during execution. All the cores in a socket share the components in the uncore (on-die logic outside of 
the cores), Figure 1. Due to the contention for the shared resources such as LLC and memory bandwidth, the performance and energy consumption of individual cores gets influenced by other cores. The level of competition for these shared resources any application encounters varies with the nature of its co-runners how hungry they are for the same resources. Consequently, it makes it hard to predict performance-energy trade-off and operate DVFS in a multicore environment.

In the Intel Sandy Bridge and Ivy Bridge generations, all the cores (including their private caches) and the uncore sections (which contains the Chip Interconnect (CI), Last Level Cache (LLC), Memory Controllers (MC), etc.) of the processor share a single variable voltage and frequency domain (Gough et al. 2015). When the operating system sets a particular voltage and frequency for the cores, it applies to the whole chip. However, in recent architectures, Intel has released Xeon processors (Intel Haswell-EP, Broadwell-EP, etc.) with separate voltage and frequency domains for individual cores enabling them to support per-core DVFS. In these processors (see Figure 1), the uncore also has separate variable voltage and frequency domain so that it is possible to control it independently (Gough et al. 2015) and improve energy efficiency. However, predicting the impact of frequency and voltage scaling on the performance and energy consumption of applications that are interacting with each other is now non-trivial. For an $n$-core processor with $m$ DVFS frequency choices, the total DVFS configuration space is now $(m)^{n+1}$ (with the uncore accounting for the + 1). In these systems, any core DVFS decision by the frequency governor not only affects the performance of the application which runs on that particular core but also has an indirect impact (typically smaller) on others. On the other hand, since all the cores share the uncore, uncore frequency scaling (UFS) is more likely to substantially impact the performance of all the application programs currently running on all the cores. In this paper, we model and predict the performance-energy trade-off using a learning-based UFS.

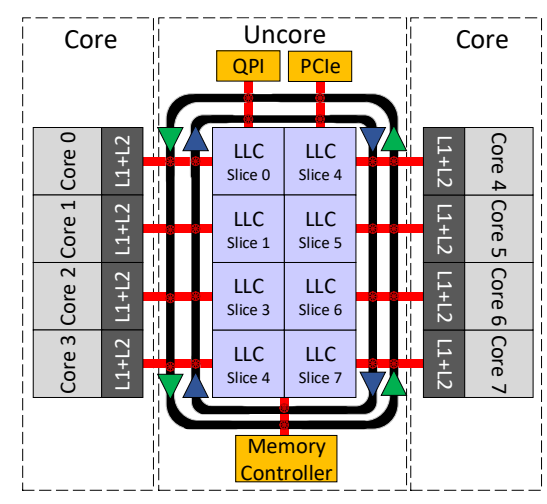

Figure 1: Core and uncore in modern multicore processors.

We assume that applications are currently running on the available cores at preset core frequencies. When the uncore frequency scales down, it affects the performance of the applications differently. Some slowdown significantly while others see a negligible impact. The impact on the performance of the application program running on a specific core depends not only on its characteristics (its CPU utilization, memory access behavior, etc.) but also on the characteristics of its current co-runners.

The proposed machine learning based UFS model takes performance metrics collected from solitary runs (solo-runs) of applications as input and predicts the optimal uncore frequency and the associated reduction in chip-energy consumption compared to the maximum frequency run. It attempts to satisfy a QoS policy - a user-specified maximum acceptable performance loss - when deciding the uncore frequency. In our approach, such an objective implies ensuring a certain quality of service (QoS) while saving energy. The rest of the paper is organized as follows: Section 2 discusses the overall process of the model construction, and Section 3 presents the evaluation of the proposed model and its efficiency. Section 4 covers previous related works. Finally, Section 5 concludes the paper. 


\section{OVERVIEW}

As we mentioned earlier, in modern processors, core and uncore have separate voltage/frequency domains paving the way for the uncore to have UFS capability. Even though these processors have the UFS capability, by default, the uncore frequency is set indirectly as a part of BIOS settings either to the maximum of the available uncore frequencies or match the frequency of the fastest core. We argue that these schemes are energy inefficient as they lack the flexibility to exploit energy saving opportunities that arise from the varying characteristics of application programs. We perform experiments to verify the default uncore scaling scheme

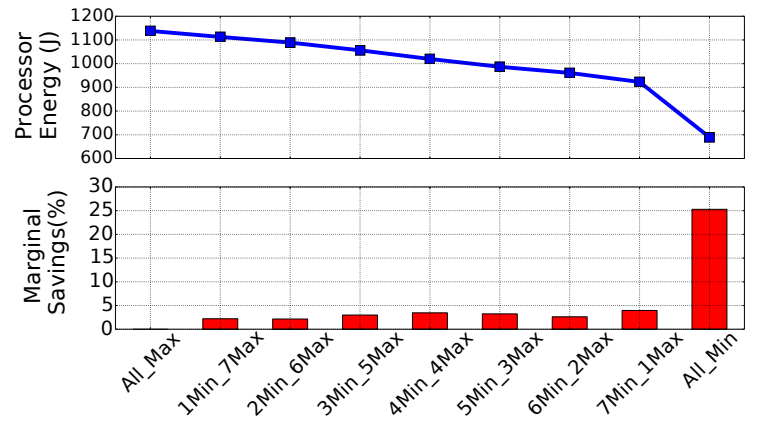

(a) Marginal processor energy savings by scaling each core from $2.1 \mathrm{GHz}$ to $1.2 \mathrm{GHz}$.

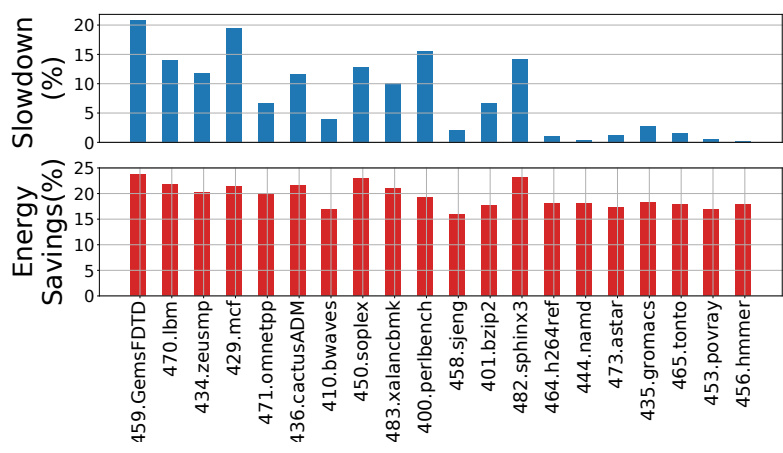

(b) Effect of UFS on performance and processor energy.

Figure 2: The default uncore frequency setting and impact of UFS on performance and energy.

of our experimental setup: Intel Xeon Processor E5-2620 v4. On this system, eight computing cores are sharing 20MB LLC. We run eight copies of namd benchmark from SPEC2006 suit. We started by setting all of the core frequencies to the highest available frequency, which is $2.1 \mathrm{GHz}$ (the turbo-boost feature is switched-off). In Figure 2a, we represent this as "All_Max". We run the programs for 30 seconds and record the energy consumption of the processor. Then we change the frequency of one of the eight cores to the lowest setting, which is $1.2 \mathrm{GHz}$, and run the experiment. We represent this as "1Min_7Max". We keep on scaling down the frequency of one additional core at a time and record the energy consumption and the marginal percentage of energy saving compared to the previous step. Finally, we go from "7Min_1Max" (in which seven cores are running at $1.2 \mathrm{GHz}$ and one core at $2.1 \mathrm{GHz}$ ) scenario to "All_Min" by scaling the only core that was running at highest frequency to the lowest. As we can see from Figure 2a, for the first seven cores, the average marginal energy saving acquired by scaling down the frequency of a single core from $2.1 \mathrm{GHz}$ to $1.2 \mathrm{GHz}$ is under $3 \%$. However, we see a $25 \%$ marginal energy saving when we scale down the last core to $1.2 \mathrm{GHz}$. This is because the uncore frequency was matching the highest core frequency -2.1 $\mathrm{GHz}$ - till the last core switched to the low frequency. Here, we can see that the default uncore setting can be energy inefficient for applications whose performance is highly dependent on core frequency (computebound applications). We also performed another set of experiments on 20 SPEC2006 applications. We ran eight copies of each application on the eight cores at maximum core frequency and scaled the uncore from maximum to minimum. We plotted the performance degradation and the associated energy saving. As we can see in Figure 2b, the energy saving achieved by different benchmarks varies in a small range between $16 \%$ to $24 \%$. However, the associated performance overhead incurred by these benchmarks differs significantly. Some of the applications save up to $18 \%$ processor energy with less than $1 \%$ of slowdown while others save nearly the same amount of energy by incurring around $20 \%$ slowdown. Therefore, a fast and accurate prediction mechanism is a necessity to perform a trade-off (performance-energy) analysis before applying UFS. 
In this Section, first, we discuss the characteristics that best captures the nature of a benchmark. Then we analyze the application's performance response to resource contention and core/uncore frequency scaling. Based on the findings, we present a methodology for building a learning-based model that drives the relationship among the application characteristics, the available uncore frequency levels, the performance loss compared to the maximum frequency run and the associated energy saving.

\subsection{Contention In CMPs}

In order to construct a model that chooses the optimal uncore frequency for applications running on the cores, we first choose the right performance metrics that capture applications' characteristics especially in relation to the potential contention that exists when they share resources. We present two light-weight performance metrics that capture the level of resource contention. The first metric, A_Score - Aggressiveness Score - of an application, captures the resource hungriness of the application. The second metric, S_Score - Sensitivity Score - of an application, represents how sensitive the application is to resource contention.

\subsubsection{A_Score}

The slowdown encountered by individual applications depends considerably on the set of co-runners it runs with at any instance. The more the application slows down, the more insensitive it will be to frequency change and vice versa. Therefore, the effect of uncore frequency scaling also depends on the co-runners. In (Abera et al. 2018), we collected various performance metrics and tested their ability to predict the potential contention among the co-running applications. We showed LLC misses per 1K cycles (MPKC) is the most correlated metric with slowdown. In this work also we use MPKC as $A \_S c o r e$ and to model the global resource pressure emanating from the co-runners, we define global-aggressiveness ( $G A$ ) as the sum of the individual $A \_$Scores of the co-runners.

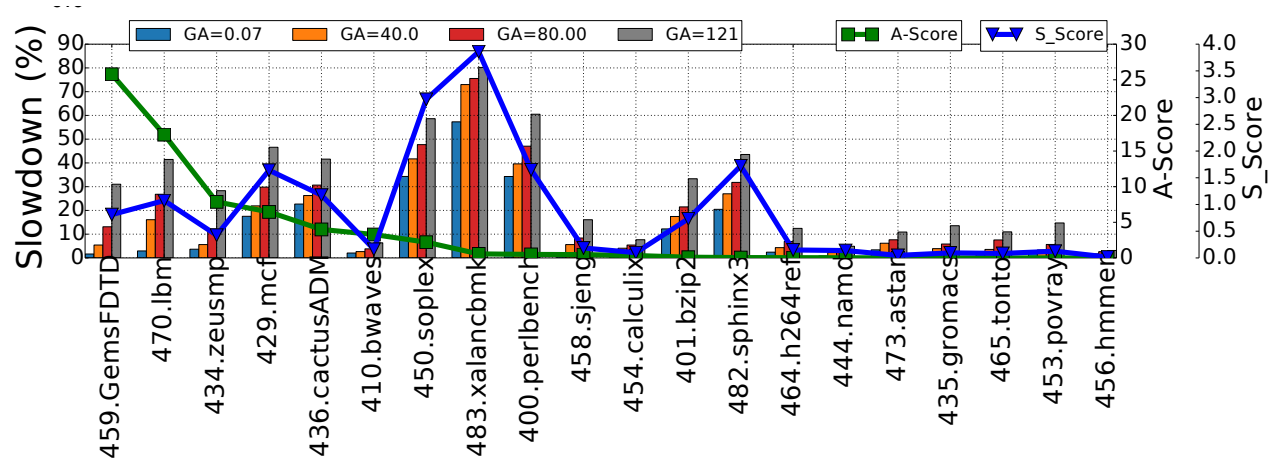

Figure 3: Impact of contention on performance.

We performed co-run experiments on 20 SPEC2006 applications. We ran each of the benchmarks with four sets of co-runner groups (each set having seven benchmarks running on the other cores of the CPU). The four co-runner groups pose different global-aggressiveness scores ranging from 0.07 to 121 (i.e., $G A=0.07, G A=40, G A=80$ and $G A=121$ ). The solo-run of each application is used as a reference to quantify the performance degradation they face in the wake of competition from the mentioned co-runner groups. We plotted the percentage slowdown encountered by each application for their runs with the co-runner groups. Along with that, we plotted the A_Score of each of the benchmark. As can be seen in Figure 3, applications with low A_Score are not affected by the contention while applications at the left extreme (memory-bound) show higher degradation. However, applications such as soplex, xal ancbmk, perlbench, sphinx 3 and bzip2 show different behavior. Even though these applications have low A_Scores, they showed highperformance degradation because of the contention from the co-runners. From this, we can see that A_Score will not be enough to accurately capture the potential performance slowdown that applications incur in a corun environment. 


\subsubsection{S_Score}

For the same level of pressure posed by the co-runners, different benchmarks are affected differently. Some of them increase their execution time by a significant margin, while others do not. As we mentioned in the previous section, the A_Score correlates well in the case of compute-intensive and bandwidth-intensive applications. However, cache-friendly applications that generally have low A_Scores, showed higher slowdowns. This is because that the A_Score value is a solo-run reading and in a solo-run environment cache-friendly applications show smaller cache misses because of their data locality. When they share their cache space with others, they show entirely different behavior as they have to use the bus more often. Therefore, we need to have an additional metric that characterizes such applications.

We examined applications' response to effective cache space by partitioning the cache. We use Intel's cache allocation technology (CAT) to perform this experiment. We partitioned the $20 \mathrm{MB}$ (20-way) cache at a granularity of $1 \mathrm{MB}$ (way-based cache partitioning). We start the experiment by allocating a $1 \mathrm{MB}$ cache to the application and record its performance. Then we keep increasing the cache by $1 \mathrm{MB}$ till we reach $20 \mathrm{MB}$. We calculated the slowdown at each partition compared to the base run - the run with the entire $20 \mathrm{MB}$ allocated to the application.

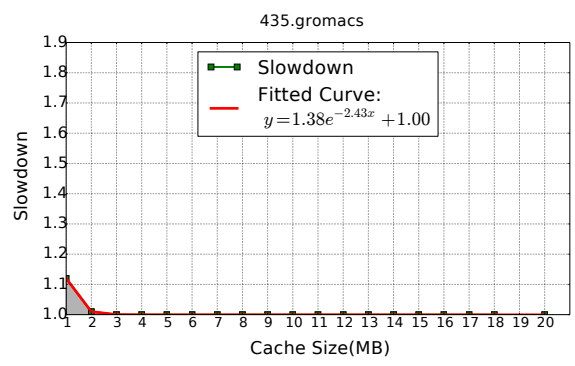

(a) gromacs.

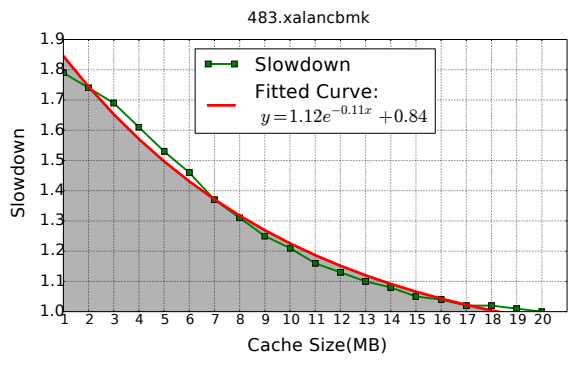

(b) xalancbmk.

Figure 4: Effect of cache space on performance.

We plot the slowdown vis-à-vis the cache sizes of applications from SPEC2006 suit. As we can see in Figure 4, the curves exhibit an exponential decay trend. Some saturate fast while others show a steady drop till it reaches the 20MB mark. Small cache size can be enough for applications with high temporal locality making them more protected against contention. gromacs - Figure 4a, shows this behavior with a fast dropping and saturating curve. On the other hand, there are applications which benefit from additional cache space till the end, e.g., Figure $4 \mathrm{~b}$. For these applications, even smaller competition from co-runners would be reflected in their performance, making them very sensitive to shared cache space. Their operating point slides along the curve depending on the level of global aggressiveness they encounter $(G A)$. Along with the slowdown curve, we plotted its exponential fit curve to show the correlation, Figure 4 . The behavior manifested in the plots can be used to differentiate the cache-sensitive applications from the others. We use the area under the exponential fit curve, depicted as a shade in Figure 4, to approximate the cache sensitivity of applications. We use the integral of the fit_curve to represent S_Score of the applications.

$$
\text { S_Score }=\int_{x=1}^{x=20}\left(a e^{-b x}+c\right) d x .
$$

As we can see from (1), S_Score of an application is represented as the integral of the exponential fit curve, where $a, b$ and $c$ represent the coefficients of the fit. It showed a correlation of 0.91 with the slowdown, and it best correlates with the outliers we encountered in Figure 3. 


\subsection{Impact of Contention and Core/Uncore DVFS on Performance}

We performed motivational experiments on applications from the SPEC CPU2006 benchmark suite to understand the range of energy savings/performance loss trade-off. In these tests, we ran each benchmark on an Octa-core CMP sharing 20MB LLC cache with seven co-runner benchmarks running on the remaining cores. We prepared eight different sets of co-runners, each set having seven benchmarks, and posing a

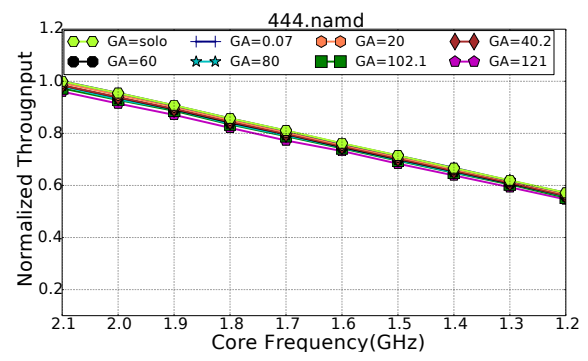

(a) Performance response of 444.namd benchmark to core frequency scaling.

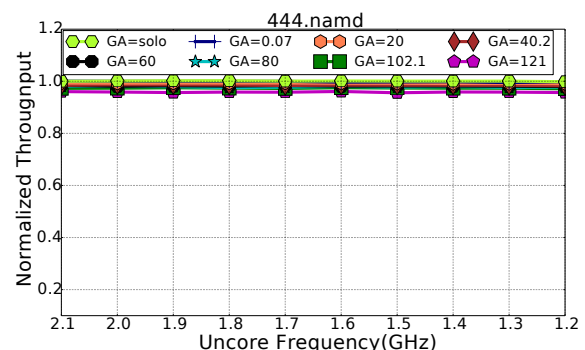

(b) Performance response of 444.namd benchmark to uncore frequency scaling.

Figure 5: Effect of Core/Uncore DVFS and contention on performance of compute-bound applications.

different cumulative pressure on the shared resources. We quantified the collective pressure of the seven co-runners by their $G A$ value. The higher the $G A$, the greater the pressure on the shared resources. One of the eight co-runner groups was an empty set - this gives us the solo-run of the application. We experimented with two scenarios. In the first, we kept the uncore at maximum frequency and scaled the core of our interest (the core where the primary application runs) from $2.1 \mathrm{GHz}$ to $1.2 \mathrm{GHz}$ with a step size of $100 \mathrm{MHz}$. In the second scenario, we kept the core at maximum frequency and scaled down the uncore from $2.1 \mathrm{GHz}$ to 1.2 $\mathrm{GHz}$ with a step size of $100 \mathrm{MHz}$. In both situations, we recorded the performance of the primary application and normalized it by its solo-run (at maximum core and uncore frequencies) performance.

Applications show different performance responses to core and uncore frequency scaling, in the presence and absence of contention. This variation depends on the application's characteristics and its execution environment as defined by the co-runners. Compute-bound applications such as namd, which make very few accesses to LLC and lower memory hierarchy, benefit from a higher core frequency as their performance linearly correlates with speed of the cores (Figure 5a). However, they are not sensitive to UFS as they have limited bus activities, as can be seen in Figure 5b. The behavior of namd stays the same regardless it runs alone or with any of the co-runner groups. This indicates that it is largely insensitive to the change in the execution environment as its performance does not rely on shared resources.

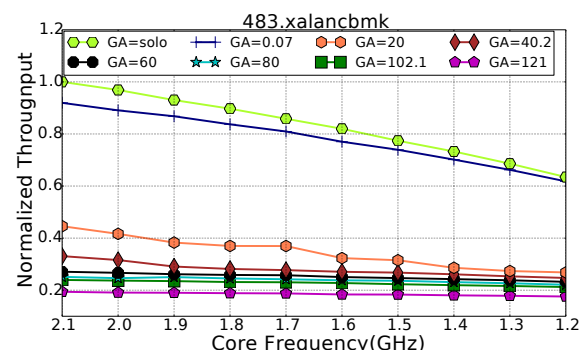

(a) Performance response of 483.xalancbmk benchmark to core frequency scaling.

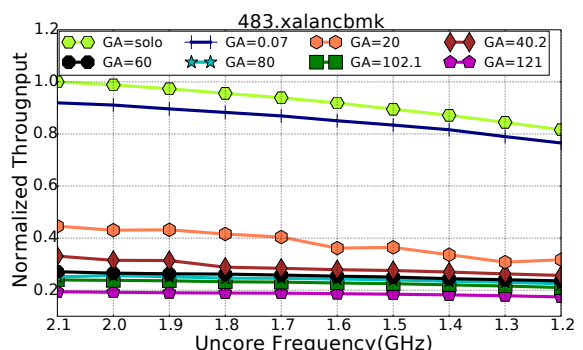

(b) Performance response of 483.xalancbmk benchmark to uncore frequency scaling.

Figure 6: Effect of Core/Uncore DVFS and contention on performance of cache-friendly applications. 
On the other hand, memory-bound applications, which make a lot of accesses to the LLC, behave differently and can broadly be divided into two classes. The first class consists of applications whose performance has a high dependence on the shared cache space: applications with high data reuse, or "cache-friendly" applications. These show higher performance when they run alone or with compute-bound applications. With such co-runners, their performance is determined by the core frequency as their memory transaction latency is hidden by the cache. In this case, their performance is also sensitive to uncore frequency scaling as they are having a high number of accesses (cache hits in particular). However, in the wake of competition for shared cache space from other memory-bound co-runners, their performance drops significantly. In such situations, the contention dominates, and increasing core/uncore frequency does not benefit much. As we can see from Figure 6, the cache-friendly benchmark xalancbomk losses its performance by more than 80 $\%$ when it runs with the most aggressive co-runner group. In this case, since the slope of the curve is so flat, we can use low core/uncore frequencies without significant performance overhead. However, when it runs alone (solo-run) and with the most compute-intensive co-runners, it shows $39 \%$ and $34 \%$ performance drop respectively, for the change in the core frequency from $2.1 \mathrm{GHz}$ to $1.2 \mathrm{GHz}$ (Figure 6a). For the same case, scaling the uncore also dents the performance by $20 \%$ and $17 \%$ respectively, as can be seen in Figure $6 \mathrm{~b}$.

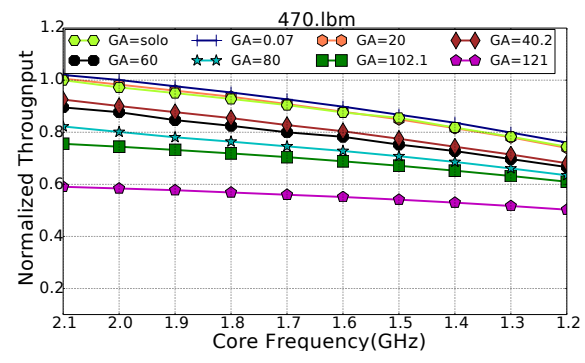

(a) Performance response of $470.1 \mathrm{bm}$ benchmark to core frequency scaling.

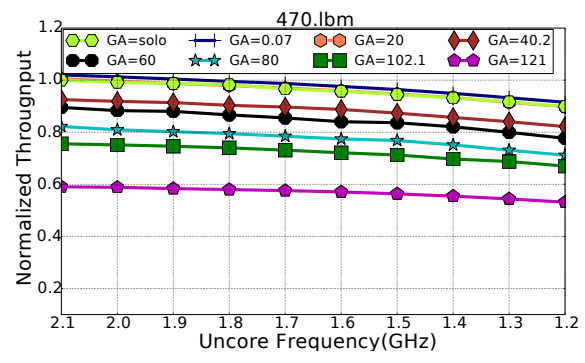

(b) Performance response of $470.1 \mathrm{bm}$ benchmark to uncore frequency scaling.

Figure 7: Effect of Core/Uncore DVFS and contention on performance of bandwidth-intensive applications.

The second class of memory-bound applications consists of bandwidth-intensives. These applications heavily use the memory bandwidth even if they run solo (without co-runners) as their streams of accesses have low temporal locality. For these applications, the impact of scaling the core frequency is much less compared to what we see in the case of compute-bound applications, as seen in Figure 7a. Scaling the uncore frequency shows a trend similar to that of the cache-friendly applications, as seen in Figure $7 \mathrm{~b}$. When they face contention from other co-runners, their performance drops, but not as much as in the case of cachefriendly applications. We analyzed 20 applications from SPEC2006 suite and the spectrum of behavior we notice lies in between these discussed extreme cases. Any DVFS policy should take these factors into account before choosing the optimal frequency at which any workload should run. In this paper, we focus on UFS as core DVFS have been covered in previous work (Abera et al. 2018).

\subsection{Data Collection}

Let us assume a processor having $N_{C}$ cores. Let us also assume we have a set of $N_{A}$ applications $A=\left\{A_{0}, A_{1}, \ldots, A_{N_{A}-1}\right\}$ that are to be run on this processor. We first collect the aggressiveness and sensitivity scores of each application $A_{\text {cur }} \in A$ as $A \_S c o r e_{A_{c u r}}$ and $S_{-} \mathrm{Score}_{A_{\text {cur }}}$ by running it alone on the given processor. We then construct a set of randomly selected $N_{C G}$ co-runner groups, $C=\left\{C G_{0}, C G_{1}, \ldots, C G_{N_{C G}-1}\right\}$, with each group having $N_{C}$ applications from the set $A$. The aggressiveness and sensitivity scores of each co-runner group $C G_{c u r}$ is represented by the vectors $A \_S c o r e_{C u r}$ and $S \_S c o r e_{C u r}$ that store the $A \_S c o r e s$ and $S \_$Scores of the applications in $C_{c u r}$. Let us also assume that the processor is capable of operating at $N c_{f}$ different core frequencies $F_{C}=\left\{f c_{0}, f c_{1}, \ldots, f c_{N c_{f}-1}\right\}$, and at $N u_{f}$ different uncore frequencies 


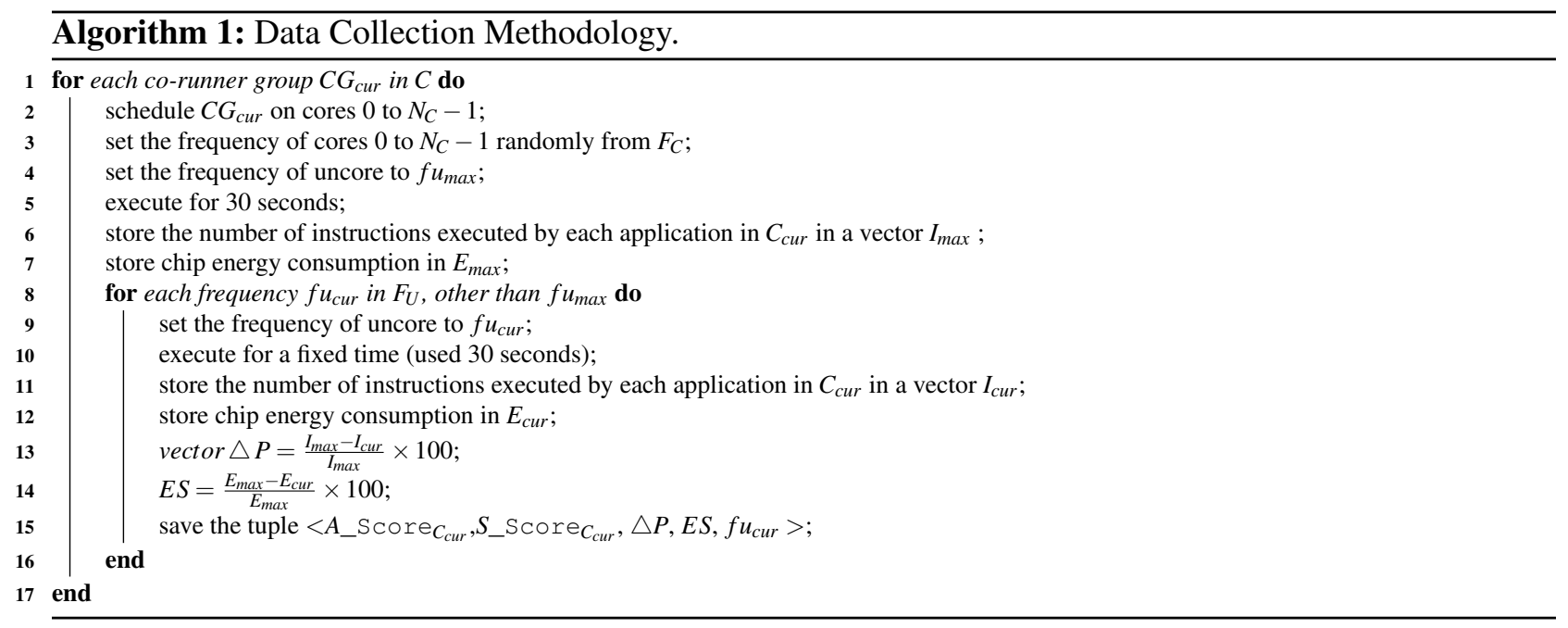

$F_{U}=\left\{f u_{0}, f u_{1}, \ldots, f u_{N u_{f}-1}\right\}$, with the maximum frequencies among these sets being labeled $f c_{\max }$ and $f u_{\max }$ respectively. Algorithm 1 describes how the data collection is done.

\subsection{UFS Predictor Model}

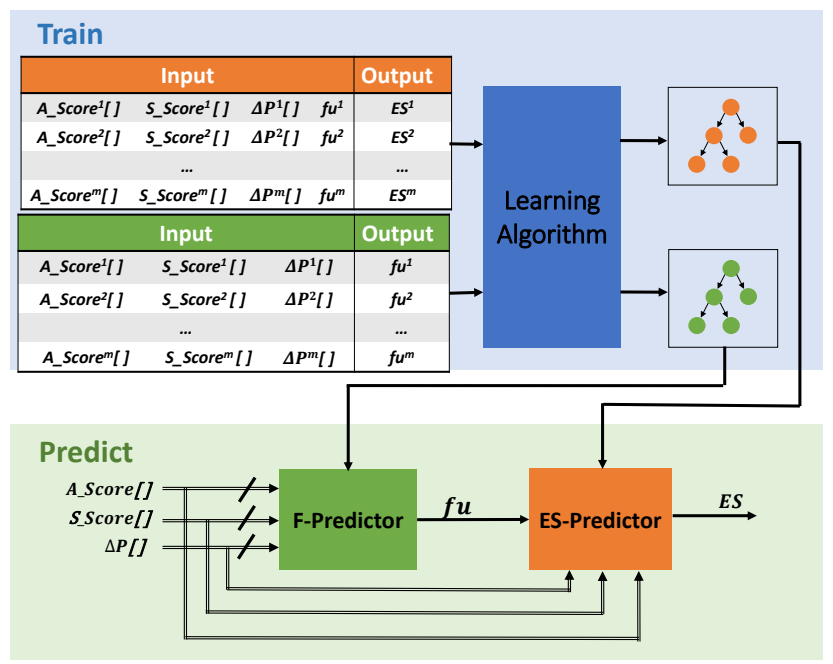

Figure 8: Training and testing of the model.

We want to develop a model that best captures the relationship between benchmarks' characteristics, the uncore frequency at which they are executed, the energy consumption and their performance. In the training phase, we use the vectors: $A \_S c o r e, S \_S c o r e, \triangle P$ of the running applications and uncore frequency at which they run (as collected in Section 2.3) to build the model, as shown in Figure 8. We build two separate models: the F-Predictor predicts the optimal uncore frequency given the $A \_S c o r e, S \_S c o r e, \triangle P$ of the applications. The ES-Predictor uses the output of the F-Predictor as an additional feature and predicts the expected energy saving (ES) that can be achieved with this target frequency. This provides the necessary performance-energy trade-off information for the UFS.

The model can be used as part of batch scheduler. We first collect per application metrics and these metrics can be used every time the user includes this application in the batch. During runtime, the user specifies the batch along with the performance requirements. The scheduler employs the proposed model and the per-application metrics, and appropriately sets the uncore frequency to maximize energy savings. 
Bekele, Balakrishnan and Kumar

\section{EVALUATION}

For the evaluation, we use applications from SPEC CPU2017 benchmark suite (Bucek et al. 2018). In each of the experiments, we execute the programs for 30 seconds. We use perf interface to read performance readings. All our experiments were performed on a dual socket Intel Xeon Processor E5-2620 v4 server system ( see Table 1). In the experiments, the hyper-threading and turbo-boost features were switchedoff. To minimize the run-to-run variations, we isolated the cores in Socket 0 from the kernel scheduler using isolcpus kernel command and use taskset to pin programs to cores. In addition to that, we repeat each experiment five times and take the average reading to further minimize run-to-run variations. For energy readings, we use Intel Running Average Power Limit (RAPL) interface. We use the cpufreq driver in "userspace" governor mode to set the CPU to a specific frequency. In addition to this, these processors come with uncore frequency scaling (UFS) capabilities that enable the programmer to set the speed of the uncore to one of the available frequencies. The uncore frequency can be set by writing to the lower 16-bits of the Model-Specific Register (MSR) 0X620.

Table 1: System Configuration.

\begin{tabular}{|c|c|c|c|}
\hline Parameters & Configuration & Parameters & Configuration \\
\hline Number of CPUs & 2 & Caches & L1I :32 KB, 8-way, per core \\
\hline $\mathrm{CPU}$ & Intel Xeon Processor E5-2620 v4 & & L1D: $32 \mathrm{~KB}, 8$-way, per core \\
\hline Cores per CPU & 8 & & L2: $256 \mathrm{~KB}, 8$-way, per core \\
\hline Threads per Core & 2 & & L3: $20 \mathrm{MB}, 20$-way, per CPU \\
\hline Frequency & Base $2.1 \mathrm{GHz}$ & OS & Linux kernel 4.16 \\
\hline Core Frequencies & $\{1200,+100, \ldots, 2100\} \mathrm{MHz}$ & Uncore Frequencies & $\{1200,+100, \ldots, 2700\} \mathrm{MHz}$ \\
\hline
\end{tabular}

\subsection{Machine Learning Algorithms}

There are a variety of machine learning algorithms to choose from. We used the WEKA - Waikato Environment for Knowledge Analysis - machine learning suite (Hall et al. ) to study the effectiveness of the different modeling alternatives. Table 2 shows the list of various machine learning algorithms with their respective correlation indices when tested using 10 fold cross-validation. We found that the decision tree based Random Forest ( $R F$ ) regression model best captures the relationship between the performance metrics, the uncore-frequency, and the performance degradation showing higher prediction accuracy. $R F$ (Breiman 2001) is an ensemble of decision trees where each tree depends on the values of a random vector sampled independently and with the same distribution for all trees in the forest. The reason that the RF model performs well is that it alleviates the over-fitting problem, which is common in many other regression models. Compared to the other algorithms, $\mathrm{RF}$ takes longer training time, but that is a one-time cost.

Table 2: Algorithm Comparison.

\begin{tabular}{l|c|c|c} 
Algorithms & Cross-Validation Score & Training time(sec) & Test time per data point(sec) \\
\hline Linear Regression & 0.9624 & 1.21 & 1.26 E-05 \\
MLPRegressor & 0.9937 & 1.47 & 5.8 E-05 \\
REPTree & 0.9924 & 0.75 & 3.8 E-05 \\
Random Forest & 0.9961 & 2.21 & $8.3 \mathrm{E}-05$
\end{tabular}

\subsection{Experiments}

We used 12 benchmarks from SPEC2017 benchmark suite as set $A=\left\{619.1 b m \_s, 549\right.$. fotonik3d_r, 620.om$n e t p p \_r$, 502.gcc_r, 510.parest_r, 523.xalancbmk_r, 625.x264_s, 638.imagick_s, 505.mcf_r, 541.leela_r, 511.povray_r, 648.exchange2_s $\}$. The benchmarks are selected based on their $A \_S c o r e s$, such that they cover the whole spectrum of $A \_$Scores observed in SPEC2017. First, we ran each of these applications in a solitary environment to collect their contention metrics: $A \_$Score and $S \_$Score. Then from set $A$, we prepared all possible (495) co-runner groups, each containing eight benchmarks. We use these co-runner 
groups for the purpose of model building, that is, set $C$ as defined in Section 2.3. The model was based on the decision tree based Random Forest $(R F)$ regression model. To test our models, we perform 10 -fold cross-validation using the training set of the co-runner groups. Since the output of the model is a real number, we approximate the value to the nearest frequency setting. We present experiments spanning five QoS policies: $1 \%, 5 \%, 10 \%, 15 \%$ and $30 \%$, and study the energy savings and performance (instructions per second) degradation in each case.

\subsection{Results and Analysis}

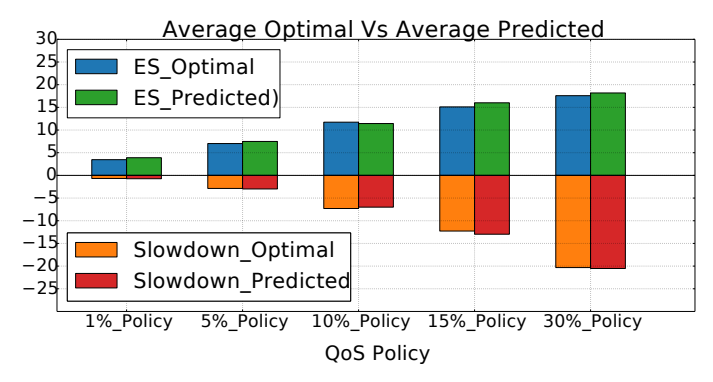

(a) Average optimal and predicted energy saving and slowdown.

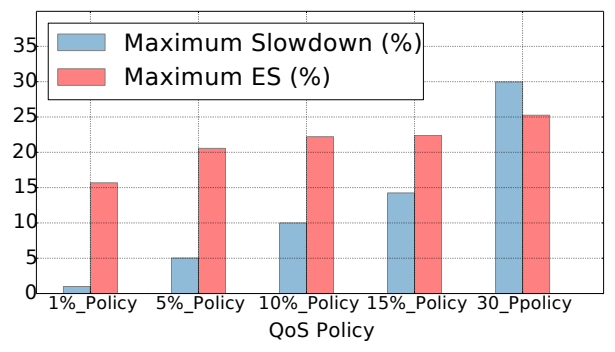

(b) Maximum energy saved with slowdown for each policy.

Figure 9: Evaluation result.

As we can see from Figure 9a, the uncore frequency predicted by our models is near-optimal - the average energy savings is within $3.6 \%$ of the optimal, while the performance degradation is within $1.7 \%$. Additionally, our predictor resulted in a QoS violation in only $7.5 \%$ of the instances. Further evaluating our models using traditional ML metrics, we observed a high correlation index of 0.9961 for the F-Predictor with average prediction accuracy of $84.9 \%$. Out of the inaccurate predictions $94 \%$ were within a distance of one frequency step (100 MHz). The ES-Predictor demonstrated a correlation index of 0.9973. The higher accuracy achieved in the ES-predictor is because of the use of the additional feature - uncore frequency as an input.

\subsubsection{Scope of Uncore Frequency Scaling}

For the one percent policy, we see that it is possible to achieve energy savings of up to $15 \%$. The average slowdown and energy saving achieved for the same policy are $0.67 \%$ and $3.46 \%$ respectively. For the 30 percent policy, we see up to $25.24 \%$ energy saving by committing $29 \%$ performance and average slowdown and energy saving of this policy is $20.32 \%$ and $17.57 \%$ respectively. This shows the diminished return of energy saving when we allow higher percentage of performance. This is because the energy saving that can be achiever by uncore frequency scaling lies in narrow band. The large difference between the average and the maximum energy savings and performance degradation indicates a high dependence on the nature of the benchmarks, as already discussed in Section 2.2. Thus, our ES-Predictor can help the end-user perform a cost-benefit analysis and take a more informed scaling decision.

\section{RELATED WORK}

Since its proposal, DVFS (Weiser et al. 1994) has been used to control the performance and energy consumption of processors through adjustment of clock frequency and supply voltage. DVFS can be applied on a per-chip, per-core or per-cluster basis and most of the previous work focused on chip-wise DVFS. There has been a significant amount of work on the use of DVFS in real-time systems to optimize energy 
consumption by utilizing slack times (Zhu et al. 2003, Kim et al. ). In another trend, machine learning algorithms have been proposed to perform intelligent DVFS based energy saving (Dhiman and Rosing 2007, Curtis-Maury et al. 2008, Cochran et al. 2011, Yang et al. 2015, Shen and Qiu 2014, Abera et al. 2018). Other previous works focused on the use of reinforcement learning in which they took task characteristics and processor configuration to scale frequency (Juan and Marculescu 2012, u. Islam and Lin 2015, Shafik et al. 2016, Wang et al. 2017). Most of these works focused on either chip-wide or per-core DVFS. To date, few works have considered the uncore and UFS based performance-energy trade-off. The advantage of scalable uncore in a heterogeneous multicore environment investigated by (Gupta et al. 2012). When there are big and small cores in the processor, and when only the small cores are active, they downscale the uncore frequency to save energy. In other work, (Sundriyal et al. 2018) studied the impact of core and/or uncore frequency scaling on scientific quantum chemistry application GAMESS. They evaluated the schemes on Intel Haswell-EP machines. In this paper, we used Intel Xeon Broadwell-EP system that allows the uncore voltage and frequency to be scaled independently of the cores. The proposed model helps not only in assisting the selection of appropriate uncore frequency but also predicts the performance-energy trade-off to support UFS decision making.

\section{CONCLUSION}

In modern processors, the computing cores and uncore are designed with separate voltage/frequency domains providing a platform for these chips to have UFS capability. Even though these features are available, still the uncore frequency and in turn, power is set as part of BIOS settings either to the maximum available uncore frequency or match the frequency of the fastest core. In this work, we showed that these approaches are energy inefficient. We also demonstrated that, given applications' memory behavior represented using a couple of lightweight parameters, we could accurately predict the optimal uncore frequency and the associated energy savings for a given upper limit on performance loss. The results demonstrated that on an average, $85 \%$ of the predictions by frequency-predictor were accurate and out of the inaccurate predictions $94 \%$ were within a distance of one frequency step (100 MHz). We also observed up to $15 \%$ of energy saving by only committing under $1 \%$ of performance.

\section{REFERENCES}

Abera, S., M. Balakrishnan, and A. Kumar. 2018. "Performance-Energy Trade-off in CMPs with Per-Core DVFS". In ARCS 2018.

Breiman, L. 2001. "Random Forests”. Machine Learning.

Bucek, J., K.-D. Lange, and J. v. Kistowski. 2018. "SPEC CPU2017: Next-Generation Compute Benchmark". In Companion of the 2018 ACM/SPEC ICPE' 18.

Cochran, R., C. Hankendi, A. K. Coskun, and S. Reda. 2011. "Pack amp; Cap: Adaptive DVFS and thread packing under power caps". In 2011 44th MICRO.

Curtis-Maury, M., A. Shah, F. Blagojevic, D. S. Nikolopoulos, B. R. de Supinski, and M. Schulz. 2008. "Prediction models for multi-dimensional power-performance optimization on many cores". In PACT.

Dhiman, G., and T. S. Rosing. 2007. "Dynamic voltage frequency scaling for multi-tasking systems using online learning”. In Proceedings of ISLPED '07.

Gough, C., I. Steiner, and W. A. Saunders. 2015. Energy Efficient Servers.

Gupta, V., P. Brett, D. Koufaty, D. Reddy, S. Hahn, K. Schwan, and G. Srinivasa. 2012. "The Forgotten 'Uncore': On the Energy-Efficiency of Heterogeneous Cores". In Presented as part of the 2012 USENIX Annual Technical Conference (USENIX ATC 12).

Hall, M., E. Frank, G. Holmes, B. Pfahringer, P. Reutemann, and I. H. Witten. "The WEKA Data Mining Software: An Update". SIGKDD Explor. 
Juan, D.-C., and D. Marculescu. 2012. "Power-aware Performance Increase via Core/Uncore Reinforcement Control for Chip-multiprocessors". In Proceedings of ISLPED ' 12.

Kim, S. I., H. T. Kim, G. S. Kang, and J.-K. Kim. "Using DVFS and Task Scheduling Algorithms for a Hard Real-time Heterogeneous Multicore Processor Environment". In Proceedings of the 2013 Workshop on Energy Efficient High Performance Parallel and Distributed Computing.

Shafik, R. A., S. Yang, A. Das, L. A. Maeda-Nunez, G. V. Merrett, and B. M. Al-Hashimi. 2016. "Learning Transfer-Based Adaptive Energy Minimization in Embedded Systems". IEEE Transactions on Computer-Aided Design of Integrated Circuits and Systems.

Sharma, Y., B. Javadi, W. Si, and D. Sun. 2016. "Reliability and Energy Efficiency in Cloud Computing Systems". J. Netw. Comput. Appl..

Shen, H., and Q. Qiu. 2014. "Contention Aware Frequency Scaling on CMPs with Guaranteed Quality of Service”. In Proceedings of DATE'14.

Sundriyal, V., M. Sosonkina, B. M. Westheimer, and M. Gordon. 2018. "Comparisons of Core and Uncore Frequency Scaling Modes in Quantum Chemistry Application GAMESS". In Proceedings of HPC'18.

u. Islam, F. M. M., and M. Lin. 2015. "A Framework for Learning Based DVFS Technique Selection and Frequency Scaling for Multi-core Real-Time Systems". In 2015 IEEE 17th HPCC.

Wang, Z., Z. Tian, J. Xu, R. K. V. Maeda, H. Li, P. Yang, Z. Wang, L. H. K. Duong, Z. Wang, and X. Chen. 2017. "Modular reinforcement learning for self-adaptive energy efficiency optimization in multicore system". In 22nd ASP-DAC.

Weiser, M., B. Welch, A. Demers, and S. Shenker. 1994. "Scheduling for Reduced CPU Energy". In Proceedings of the 1st USENIX Conference on Operating Systems Design and Implementation, OSDI '94.

Yang, S., R. A. Shafik, G. V. Merrett, E. Stott, J. M. Levine, J. Davis, and B. M. Al-Hashimi. 2015. "Adaptive energy minimization of embedded heterogeneous systems using regression-based learning". In 25th PATMOS.

Zhu, D., R. Melhem, and B. R. Childers. 2003. "Scheduling with dynamic voltage/speed adjustment using slack reclamation in multiprocessor real-time systems". IEEE Transactions on Parallel and Distributed Systems.

SOLOMON ABERA BEKELE is a Ph.D. scholar at Indian Institute of Technology Delhi (IIT Delhi). He received his Masters Degree in Computer Science and Engineering from IIT Delhi in 2012. His research interests focus on performance-energy optimization in high performance computers and embedded systems, from resource contention perspective. His email address is solomon@cse.iitd.ac.in.

M BALAKRISHNAN is a Professor in the Department of Computer Science and Engineering at Indian Institute of Technology Delhi (IIT Delhi). His research interests include Assistive Technologies, Embedded System Design, EDA and System Level Design and FPGA based Accelerators. His email address is mbala@cse.iitd.ac.in.

ANSHUL KUMAR is a Professor in the Department of Computer Science and Engineering at Indian Institute of Technology Delhi (IIT Delhi). His research interests include Embedded System Design, Hardware Software CoDesign,Application Specific Processor Synthesis, VLSI Design Automation and Computer Architecture. His email address is anshul@cse.iitd.ac.in. 